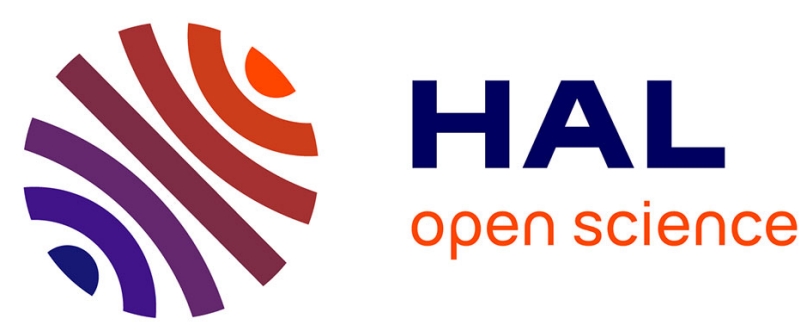

\title{
The combination of a poly-caprolactone/nano-hydroxyapatite honeycomb scaffold and mesenchymal stem cells promotes bone regeneration in rat calvarial defects
}

Marie Naudot, Alejandro Garcia Garcia, Nicolas Jankovsky, Anaïs Barre, Luciane Zabijak, Soufiane Zakaria Azdad, Louison Collet, Fahmi Bedoui, Anne Hébraud, Guy Schlatter, et al.

\section{- To cite this version:}

Marie Naudot, Alejandro Garcia Garcia, Nicolas Jankovsky, Anaïs Barre, Luciane Zabijak, et al.. The combination of a poly-caprolactone/nano-hydroxyapatite honeycomb scaffold and mesenchymal stem cells promotes bone regeneration in rat calvarial defects. Journal of Tissue Engineering and Regenerative Medicine, 2020, 10.1002/term.3114 . hal-02961861

\section{HAL Id: hal-02961861 https://hal.science/hal-02961861}

Submitted on 14 Dec 2020

HAL is a multi-disciplinary open access archive for the deposit and dissemination of scientific research documents, whether they are published or not. The documents may come from teaching and research institutions in France or abroad, or from public or private research centers.
L'archive ouverte pluridisciplinaire HAL, est destinée au dépôt et à la diffusion de documents scientifiques de niveau recherche, publiés ou non, émanant des établissements d'enseignement et de recherche français ou étrangers, des laboratoires publics ou privés. 


\section{The combination of a poly-caprolactone/nano-}

\section{hydroxyapatite honeycomb scaffold and mesenchymal stem cells promotes bone regeneration in rat calvarial}

\section{defects}

Running title : PCL-nHA honeycomb scaffold with MSCs promotes bone regeneration in vivo

Marie Naudot ${ }^{1}$, Alejandro Garcia Garcia ${ }^{2}$, Nicolas Jankovsky ${ }^{3}$, Anaïs Barre ${ }^{1}$, Luciane Zabijak $^{4}$, Soufiane Zakaria Azdad ${ }^{5}$, Louison Collet $^{3}$, Fahmi Bedoui $^{6}$, Anne Hébraud ${ }^{7}$, Guy Schlatter $^{7}$, Bernard Devauchelle ${ }^{1,8,9}$, Jean-Pierre Marolleau ${ }^{3,10}$, Cécile Legallais ${ }^{2}$, Sophie Le Ricousse $^{1 *}$

1: EA7516, CHIMERE. Jules Verne University of Picardie, Amiens, France

2: UMR 7338, BMBI. CNRS, University of Technology of Compiegne, Compiegne, France

3: EA4666, HEMATIM. Jules Verne University of Picardie, Amiens, France

4: Plateforme ICAP. Jules Verne University of Picardie, Amiens, France

5: Department of Pathology and Cytology, Amiens University Medical Center, Amiens,

France

6: Laboratoire Roberval, FRE CNRS 2012, University of Technology of Compiegne,

Compiegne, France

7: ICPEES UMR 7515. CNRS, University of Strasbourg, Strasbourg, France

8: Department of Maxillofacial Surgery, Amiens University Medical Center, Amiens, France

9: Facing Faces Institute, Amiens, France,

10: Department of Hematology, Amiens University Medical Center, Amiens, France 
* Correspondence: sophie.le.ricousse@u-picardie.fr, Tel.: +33-322-825-396

\section{Abstract:}

Bone tissue engineering goes beyond the limitations of conventional methods of treating bone loss, such as autograft-induced morbidity and a lack of integration for large grafts. Novel biomimicry approaches (using three-dimensional [3D] electrospinning and printing techniques) have been designed to offer the most appropriate environment for cells and thus promote bone regeneration. In the present study, we assessed the bone regeneration properties of a composite 3D honeycomb structure from the electrostatic template assisted deposition (ETAD) process by an alternate deposition of electrospun polycaprolactone (PCL) nanofibers and electrosprayed hydroxyapatite nanoparticles (nHA) on a honeycomb micropatterned substrate. We first confirmed the biocompatibility of this honeycomb PCL-nHA scaffold in culture with bone-marrow-derived mesenchymal stem cells (MSCs). The scaffold was then implanted (alone or with seeded MSCs) for 2 months in a rat critical-sized calvarial defect model. The observation of new bone synthesis in situ (monitored using micro-computed tomography every 2 weeks and a histological assessment upon extraction) demonstrated that the honeycomb PCL-nHA scaffold was osteoconductive. Moreover, the combination of the scaffold with MSCs was associated with significantly greater bone volume and mineralized regeneration during the 2-month experiment. The combination of the biomimetic honeycomb PCL-nHA scaffold with patient stem cells might therefore have great potential for applications in maxillofacial surgery.

Keywords: biomaterials - electrospinning - mesenchymal stem cell - bone regeneration calvarial defect - honeycomb 


\section{Introduction}

Over the last few decades, tissue-engineered bone substitutes have been developed as alternatives to autografts and allografts; bone grafts are often only available in limited quantities, and are associated with a risk of donor site morbidity and infection. The use of an appropriate biomaterial-based scaffold is crucial for accurately restoring, maintaining and improving the bone tissue regeneration process in space and time. The scaffold has to provide an architectural blueprint for cell regeneration and must possess osteoconductive, osteoinductive and/or osteointegration properties. A number of efficient scaffolds have been developed from natural or synthetic polymers and ceramics. The most commonly used synthetic polymers are poly ( $\alpha$-hydroxyesters) such as poly( $\varepsilon$-caprolactone) (PCL), polylactic acid and poly(lactic-co-glycolic) acid (PLG). The poly ( $\alpha$-hydroxyesters)' architecture (porosity, degradability, and pore size) and mechanical properties can be tailored for specific applications, including bone tissue engineering (Borden et al., 2003; Mankani et al., 2001; Yao et al., 2019). Although PCL has a slow biodegradation rate and a high osteointegration capacity (Rao et al., 2018), it suffers from a lack of biological functionality at the scaffoldcell interface. Hence, PCL is typically combined with other natural or synthetic polymers and/or with ceramics (Shor et al., 2009; Xu et al., 2010). Bioceramics like hydroxyapatite (HA) and tricalcium phosphate (TCP) have been widely used in bone repair, in view of their chemical similarity to bone. In particular, HA mechanically reinforces other polymers and improves the scaffold's osteoconductive and osteoinductive properties (Dorozhkin 2010; Zhang et al., 2016). Three-dimensionally printed and electrospun scaffolds made from appropriate materials therefore appear to be highly promising tools for bone regeneration, due to their ability to mimic the native extracellular matrix environment via their morphology, surface modification, and/or bulk incorporation of biomolecules (Leijten and Khademhosseini 2016; Ye et al., 2019). Electrospinning techniques can produce nano- or microfibers by 
applying a high potential voltage difference between a liquid polymer and a collecting target. Oriented or structured nanofiber mats can be prepared by adjusting the direction of the electric field and micropatterning the collector (Criscenti et al., 2017; Yang et al., 2005). Researchers have reported that such structured electrospun polymer nanofibers can induce cell differentiation in vitro, which suggests that applications in bone tissue engineering are possible (Baudequin et al., 2017; Brennan et al., 2015). Recently, more complex, bioinspired multi-layered composite scaffolds have been produced by electrospinning/electrospraying on micropatterned collectors thanks to an electrostatic template assisted deposition (ETAD) process (Wittmer et al., 2014). When using honeycomb micropatterned collectors, the resulting scaffolds with controlled pore size mimic the hemi-osteon structure (Nedjari et al., 2014). A PCL scaffold integrating HA nanoparticles with a honeycomb macrostructure (referred to henceforth as a "honeycomb PCL-nHA scaffold") proved to be biocompatible, osteoconductive and osteoinductive in vitro using the $\mathrm{C} 3 \mathrm{H} 10$ cell line and organotypic culture (Garcia Garcia et al., 2018) but has not yet been tested in vivo.

In bone tissue engineering, the cellular component is usually composed of stem cells. When seeded into a scaffold, these cells have crucial roles by secreting growth factors and/or by directly differentiating into the desired osteogenic cell type (Alford et al., 2015; Zhang et al., 2012). Mesenchymal stem cells (MSCs) are multipotent stromal cells present in most adult connective tissues and most neonatal tissues. The most commonly used MSCs are isolated from the bone marrow (BM), adipose tissue or dental pulp (Chamieh et al., 2016; Chen et al., 2019). The MSCs are able to differentiate into multiple cell types, including osteoblasts, chondroblasts and adipocytes in particular (Oryan et al., 2017; Sousa et al., 2014). These cells are known to release cell survival and proliferative factors and to have immunomodulatory properties- thus controlling inflammation and modifying nearby immune cells (Kiernan et al., 2018). The positive effect of adding MSCs to a PCL scaffold on bone regeneration has 
already been demonstrated in (for example) a rabbit knee defect model (with a PCL-TCP scaffold) (Shao et al., 2006), a rat calvarial defect model (with a PLG-HA scaffold) (Orbay et al., 2017), and a porcine critical-size bone defect model (with a PCL-TCP/HA scaffold) (Jensen et al., 2016).

In the present study, we investigated the osteocompatibility, osteoconductive and osteoinductive properties of a honeycomb PCL-nHA scaffold seeded (or not) with rat BMderived MSCs (BM-MSCs) in a rat calvarial defect (Figure 1).

We first analyzed the in vitro adhesion and viability of BM-MSCs seeded on the scaffold. In vivo bone regeneration in calvarial defects was then assessed using micro-computed tomography ( $\mu$-CT) after 2, 4, 6 and 8 weeks of implantation of the honeycomb PCL-nHA with or without BM-MSCs, and at 8 weeks (the end of experiment) using histological techniques. Our experimental protocol enabled us to evaluate the respective effects of the biomaterial itself (compared with the empty space that nevertheless follows a healing process) and the additional presence of BM-MSCs seeded prior to implantation.

\section{Materials and methods}

2.1 Synthesis of the PCL honeycomb by electrospinning and electrospraying

A $6 \%$ electrospinning solution of PCL (molecular weight=80 kg.mol ${ }^{-1}$, Sigma Aldrich, France) was obtained by dissolving the polymer in dichloromethane (Sigma-Aldrich) and N,N-dimethylformamide (ReagentPlus Sigma-Aldrich) (70/30 v/v) for $24 \mathrm{~h}$. A $10 \%$ by weight HA nanopowder (Sigma-Aldrich, ref 677418) with a particle size $\leq 200 \mathrm{~nm}$ was prepared in ethanol (Sigma-Aldrich) $48 \mathrm{~h}$ before electrospraying. The obtained nHA solution was ultrasonicated for $5 \mathrm{~min}$ (Branson Sonifier, Switzerland) just before the deposition.

We used a custom vertical electrospinning device comprising a $18 \mathrm{G}$ needle connected to a Spellman SL 10 high voltage supply, a syringe pump and a rotating collector (100 rpm) 
within which a honeycomb micropattern collector was fixed. The micropattern has been described previously (Nedjari et al., 2014). The mean diameter of the honeycomb cavities was $160 \mu \mathrm{m}$, and the honeycomb walls were $20 \mu \mathrm{m}$ wide and $60 \mu \mathrm{m}$ high. Thirteen layers of PCL (flow rate: $1.5 \mathrm{ml} / \mathrm{h}$; needle-collector distance: $18 \mathrm{~cm}$; voltage: $25 \mathrm{kV}$ ) and 13 layers of $\mathrm{nHA}$ (flow rate: $0.6 \mathrm{ml} / \mathrm{h}$; needle-collector distance: $15.5 \mathrm{~cm}$; voltage: $25 \mathrm{kV}$ ) were deposited alternately, as described previously (Garcia Garcia et al., 2018) (Figure 1A). After processing, the resulting membrane was peeled off from the honeycomb micropattern collector, transferred into 6-well inserts (diameter: $12 \mathrm{~mm}$; no membrane, Costar, Corning, France), sterilized in $70 \%$ ethanol for $1 \mathrm{~h}$, and then rinsed three times with phosphate-buffered saline (PBS, Sigma-Aldrich).

\subsection{Isolation and culture of rat BM-MSCs}

Mesenchymal stem cells were harvested from 12-week-old male Sprague-Dawley rats (Janvier Labs, France). The femurs were dissected and the BM was flushed with Eagle's minimum essential medium alpha modification ( $\alpha$ MEM, Sigma-Aldrich) supplemented with 10\% fetal bovine serum (Eurobio, France), 2 mM L-glutamine (Eurobio), $100 \mathrm{U} / \mathrm{ml}$ penicillin, $100 \mathrm{pg} / \mathrm{ml}$ streptomycin (both from Eurobio), and $250 \mathrm{ng} / \mathrm{mL}$ amphotericin B (Gibco, France). The cell suspension was transferred into 6-well cell culture plates and cultured in a humidified $5 \% \mathrm{CO}_{2}$ atmosphere at $37^{\circ} \mathrm{C}$. Thereafter, the medium was replaced twice a week to remove non-adherent cells and refresh the culture. Once $80 \%$ confluence had been reached (typically after 7 to 10 days), cells were detached (using $0.25 \%$ trypsin) and replated in T175 flasks (Sigma-Aldrich).

\subsection{Phenotyping of rat BM-MSCs}


Cultured cells were harvested, suspended in PBS, and phenotyped using flow cytometry with FITC-conjugated anti-rat CD90, FITC-conjugated anti-rat CD45 antibodies or primary antibody IgG mouse anti-CD73 and then with the secondary PE-conjugated anti-mouse IgG antibody (all purchased from BD Pharmingen ${ }^{\mathrm{TM}}$, BD Biosciences, France). Cells incubated with PE-conjugated or FITC-conjugated mouse IgG1 isotype antibodies (BD Biosciences) were used as negative controls. The cells were analyzed on a BD FACSCanto ${ }^{\mathrm{TM}}$ II (BD Biosciences) and the results were analyzed using FlowJo software (version 10, Tree Star, USA).

\subsection{Cell seeding on the scaffold}

In order to assess cell attachment on the honeycomb PCL-nHA scaffold, 1.2x10 ${ }^{6}$ BM-MSCs were seeded into scaffolds in inserts with culture medium and allowed to adhere for 24 hours at $37^{\circ} \mathrm{C}$ in a $5 \% \mathrm{CO}_{2}$ atmosphere. Subsequently, the medium was changed and non-adherent cells were removed. The inserts were examined under an SEM (Philips XL30 ESEM-FEG, FEI, The Netherlands). Scaffolds seeded (or not) with cells were cleaned with ethanol and coated with gold prior to observation. In order to evaluate cell viability in the honeycomb scaffold, the scaffolds were removed from the inserts and placed in a 24 -well plate. One hour before the assay, the medium was replaced with $\alpha$ MEM medium containing Hoechst 33342 (2 $\mu \mathrm{g} / \mathrm{ml}$ ) and ethidium homodimer 1 (EthD-1, $4 \mu \mathrm{M}$ ) (both from ThermoFisher Scientific, France). The scaffolds were then rinsed with PBS and observed under a confocal microscope (Zeiss LSM 710, 20x, France).

2.5 Surgical procedures and graft preparation in the rat critical-sized calvarial bone defect model 
All procedures for animal experiments were evaluated and approved by the local animal care and use committee and by the French Ministry of Research (registration number: APAFIS 6019-2016070615199279). Twenty-two male Sprague-Dawley rats (8 weeks of age, body weight: $\approx 350 \mathrm{~g}$, Janvier Lab) were used. Twenty-four hours after cell seeding (or not), the scaffolds were grafted into a calvarial bone lesion. Rats were anesthetized by isofluorane inhalation (induction with $5 \%$ isofluorane and an airflow of $1 \mathrm{~L} / \mathrm{min} ; 3 \%$ at $0.5 \mathrm{~L} / \mathrm{min}$ thereafter; Isovet, Piramal HealthCare, UK). The scalps covering the calvarial vault were shaved, scrubbed with an antiseptic solution (Biseptine, Bayer Healthcare, France), and infiltrated with $0.1-0.5 \mathrm{ml}$ of a local anesthetic (2\% lidocaine, Lurocaïne, Vetoquinol, France). A sagittal skin incision was made over the calvarium, the soft tissues were raised, and the periosteum was dissected to expose the calvarial bone surface. A circular bone defect (diameter: $5 \mathrm{~mm}$ ) was created on each side of the sagittal suture, using a diamond bur (Fig1.C). Honeycomb PCL-nHA scaffolds with BM-MSCs (in the scaffold+MSCs group of rats, $n=11$ ) or scaffolds alone (the scaffold group, $n=11$ ) were placed into the defects on the left side; the right side remained empty and served as a control $(n=22)$. Next, the soft tissues were lowered and skin was closed with 5-0 braided absorbable suture (Vicryl 5/0, Ethicon, France). After surgery, analgesia was provided through subcutaneous injections of buprenorphine $(0.05 \mathrm{mg} / \mathrm{kg}$, Buprecare, Axience, France). The animals were housed 1 per cage for 2 weeks post-op and then 2 per cage. They had ad libitum access to water and chow, in an enriched environment. At the study end point (8 weeks after scaffold implantation), all animals were euthanized by overdose $\mathrm{CO}_{2}$ inhalation. After skin dissection, the calvarium was removed and immediately fixed in $4 \%$ neutral buffered formalin (VWR, France).

\subsection{In vivo $\mu \mathrm{CT}$ assessment and radiomorphometric analysis}

Prior to each $\mu \mathrm{CT}$ assessment of bone regeneration, rats were anesthetized with isofluorane (induction at $5 \%$ and an airflow of $1 \mathrm{~L} / \mathrm{min}$, and then maintenance with $3 \%$ at $0.5 \mathrm{~L} / \mathrm{min}$ ). The 
animals were imaged using an X-ray $\mu \mathrm{CT}$ device (SKYSCAN 1176,; X-ray source: $65 \mathrm{kV}$, $380 \mu \mathrm{A}, 1 \mathrm{~mm}$ Alu filter, and a $0.6^{\circ}$ rotation step, Bruker, USA). Three-dimensional images were acquired with a maximum voxel size of $18 \mu \mathrm{m}$. An internal density phantom (calibrated in $\mathrm{g} / \mathrm{cm}^{3}$ of HA) was used to scale the bone density. The full 3D high-resolution raw dataset was obtained with a flat panel detector that moved $180^{\circ}$ around the sample (scanning time: 5 min). Three-dimensional renderings were extracted from data frames using Dataviewer software (Bruker, USA). The amount of regenerated bone inside each defect was quantified using CTscan Analyser software (Bruker, USA). An overall volume of interest in the defect area was drawn by interpolating two-dimensional regions of interest on consecutive sections. This volume of interest comprised only the remodeled bone defect area. Overall thresholding was used to separate mineralized elements from background noise. We analyzed the bone mineral density (BMD, in $\mathrm{g} / \mathrm{cm}^{2}$ of $\mathrm{HA}$ ) and the bone volume fraction (bone volume/tissue volume, BV/TV, expressed as a percentage). Three-dimensional images of bone were reconstructed with CTvox software (Bruker, USA).

\subsection{Histological assessments}

Harvested, fixed samples were immersed in Microdec EDTA A1 decalcification solution (Microm Microtech, France) for 4 days, dehydrated in graded ethanol solutions, embedded in paraffin, prepared as $3 \mu \mathrm{m}$ sections, and stained with hematoxylin and eosin (H\&E) reagent or Masson's trichrome reagent. The images were acquired using a Leica SCN 400 Slide Scanner (Leica, France).

\subsection{Statistical analysis:}

Quantitative variables were quoted as the mean \pm standard deviation. The statistical significance of intergroup differences was probed in a one-way ANOVA test with Dunnett's post-test for multiple comparisons. The following thresholds for statistical significance were 
considered: $* \mathrm{p}<0.05, * * \mathrm{p}<0.01$, and $* * * \mathrm{p}<0.001$. All statistical analyses were performed using GraphPad Prism software (version 6, GraphPad Software, USA).

\section{Results}

\subsection{Characterization of rat BM-MSCs}

Flow cytometry with antibodies against some specific markers commonly used to characterize human MSCs (namely CD90, CD73, and CD45) was realized to immunophenotype rat BMMSCs. The latter were positive for CD90 (99.6\%) and CD73 (54\%) and negative for CD45 $(5.36 \%)$ (Figure 2).

\subsection{Adhesion and viability of BM-MSCs on the honeycomb PCL-nHA scaffold}

In order to assess the behavior of BM-MSCs in contact with the matrix, cells were seeded on the honeycomb PCL-nHA scaffold for $24 \mathrm{~h}$ and cultured. The spatial distribution and viability of BM-MSCs on honeycomb PCL-nHA scaffold were evaluated under the SEM (Figure 3.A, 3.B) and the fluorescence microscope, respectively (Figure 3.C-E).

Scanning electron microscopy revealed that the scaffold was completely covered by several layers of cells (Figure 3.B). Indeed, the scaffold's honeycomb structure was no longer visible. The cells filled the cavities and also settled on the walls. Cell viability was assessed by Hoechst 33342 staining for total cells and EthD-1 staining for dead cells (Figure 3.C-E). Only a few dead cells were observed, and the estimated viability was 95\%. Nuclear fluorescence also testified to complete occupation of the honeycomb PCL-nHA scaffold by BM-MSCs (Figure 3.C).

3.3 Bone remodeling processes in honeycomb PCL-nHA-filled defects 
To evaluate in vivo the osteoconductive and osteoinductive properties of the honeycomb PCLnHA scaffold seeded (or not) with BM-MSCs in calvarial defects, bone regeneration were assessed using $\mu$-CT 2, 4, 6 and 8 weeks after implantation (Figure 4).

In all groups, new bone emerged against the dura mater after two weeks, and an increase over time in bone regeneration was observed. The sagittal $\mu \mathrm{CT}$ views showed signs of bone bridging from the week 4 onwards, and bone bridging was more ample in the scaffold+MSCs group (as confirmed by coronal views). Under all conditions, bone reconstruction started at the margins and bottom of the defect and then became apparent in the center.

Irrespective of the condition, a quantitative analysis of bone regeneration by $\mu$-CT revealed an increase in bone formation over time, with a plateau achieved at 6 weeks (Figure 5). At that time, we observed osteogenic activity with nearly complete closure of the defect and a bone volume fraction of approximately $80 \%$.

At 2 weeks, the bone volume fraction was significantly greater in the scaffold+MSCs group than in the other two groups (Figure 5.A). At 4 weeks, we observed a slight increase of bone volume fraction in the scaffold+MSCs group $(66.6 \pm 12.4 \%)$ compare to the scaffold group $(62.4 \pm 11.2 \%, \mathrm{p}=0.2831)$ that was significantly higher in comparison to the control group $(55.4 \pm 14 \%, \mathrm{p}=0.0468)$ (Figure 5.B). Beyond 4 weeks, there were no significant intergroup differences. Irrespective of the condition, the BMD increased over time throughout the experiment - demonstrating the mineralization/maturation of newly formed bone (Figure 5.C). At all time points, the BMD was significantly higher in the scaffold+MSCs group than in the control group (Figure 5.D).

A histological assessment revealed details of the tissue composition and bone formation in the defects (Figure 6).

In the control and scaffold groups, we observed disjointed bone formation and more fibrous connective tissue (visible as light green areas in the Masson's trichrome slides) in the defects 
(Figure 6.A). In defects filled with scaffold+MSCs, we observed greater bone mature regeneration and closure of the lesion. In all groups, we observed the typical structure of mature bone, with osteocytes (the yellow arrow in Figure 6.B) encased within bone substance and osteoblasts (the green arrow in Figure 6.B) at the borders. Vessels were visible under all conditions (black arrow). No residual material was seen within the defect region.

\section{Discussion}

Many novel biomaterials and tissue-engineered products for bone regeneration have been studied over the last decades. Electrospinning has been investigated as a means of producing a 3D matrix, with a porosity that is often limited in the absence of specific microstructuration. Although most studies with electrospun biomaterials have been performed in vitro, some of the latest in vivo studies have given promising results in terms of bone regeneration in a calvarial defect model (Wang et al., 2019; Wu et al., 2019). Several polymers can be used, and are frequently combined with a mineral material to enhance the matrix's osteopromotive properties. Garcia Garcia et al. (2018) proved the in vitro value of a biomimetic structure inspired by the honeycomb structure of the osteons by combining PCL and nHA to promote the adhesion, proliferation and osteodifferentiation of the seeded cells. This scaffold had an elastic modulus of $4 \mathrm{MPa}$, which made it easy to handle and to shape into any form - as would be required in clinical practice.

In the present study, we first confirmed the in vitro biocompatibility of this honeycomb PCLnHA scaffold seeded with rat BM-MSCs by analyzing cell adhesion, spatial distribution and viability. The cells completely covered the honeycomb PCL-nHA scaffold, and so there was no preference for a particular part of the honeycomb structure; this has previously been observed with C3H10T1/2 cells after 48 h of culture (Garcia Garcia et al., 2018). This complete coverage could be explained by the honeycomb pattern's promotion of extracellular 
matrix synthesis and upregulation of type II collagen (Eniwumide et al., 2014). Alternatively, it might be due to the high seeding density - a strategy known to have delivering more cells while preserving the latter's viability and functions in vivo (Ding et al., 2017).

We next assessed the scaffold's functional properties, i.e. its ability to promote new bone formation in a rat calvarial defect model. We created two defects per animal; one was filled with scaffold, and the other remained empty as a control. This protocol makes it possible to take into account inter-individual variability and thus increasing the statistical power.

Our $\mu \mathrm{CT}$ analysis of the defects at several time points enabled us to describe the time course of bone reconstruction. The honeycomb PCL-nHA scaffold appeared to have a significant effect on bone formation as early as the second week post-implantation, with a greater bone volume fraction and a greater BMD than in the no-scaffold control group. The bone volume fraction peaked at close to $80 \%$ at 6 weeks and remained stable at 8 weeks. This regeneration time course (a logarithmic curve) has already been described in the same rat model with a beta-TCP (Chou et al., 2014) and with nanogel-crosslinked materials (Charoenlarp et al., 2018), although the investigators did not test cell-seeded scaffolds. In contrast, other studies have showed that bone reconstruction is linear (with a mean bone volume fraction of only $20 \%$ at 8 weeks) when 3D-printed PCL scaffolds were implanted in calvarial defects (Lee et al., 2019; Pae et al., 2019). The disparities between our present results and the literature data might be due to differences in the materials' composition and/or architecture. To the best of our knowledge, a PCL electrospun scaffold (with or without seeded cells) has not previously been studied with $\mu \mathrm{CT}$ at several time points (rather than at sacrifice only). We confirmed that the honeycomb PCL-nHA scaffold was osteoinductive in vivo, and that it becomes involved in the bone regeneration process soon after implantation.

As reported previously (Chamieh et al., 2016; Jensen et al., 2016; Orbay et al., 2017), bone regeneration was enhanced when the scaffolds were seeded with MSCs. Our in vivo 
densitometry data evidenced a significantly greater bone volume fraction in the defect for the scaffold+MSCs group (compared with the control group) 2 and 4 weeks after implantation. Furthermore, the BMD in the scaffold+MSCs group was higher than the control value through the experiment. However, the quantitative bone tissue regeneration observed in scaffold+MSCs group did not differ significantly from that observed with the scaffold alone. A histological assessment confirmed the $\mu \mathrm{CT}$ results; there was more bone tissue in scaffold+MSCs group, and the junction had closed completely. Hence, the addition of MSCs to a honeycomb PCL-nHA scaffold appeared to accelerate the bone healing process by increasing bone synthesis and mineralization.

It is thought that MSCs act through both direct bone formation and indirect paracrine effects that promotes extracellular matrix deposition and angiogenesis (Yagyuu et al., 2012). In our experiments, we could not identify the origin of the cells in the new bone structures, since the BM-MSCs were isolated from syngeneic rats of the same sex. Hence, we cannot conclude whether the observed bone formation was attributable to paracrine/trophic effects and/or the direct engraftment of transplanted cells.

The ideal biodegradable material should provide sufficient support for cell adhesion while leaving space for tissue growth (Kwon et al., 2018; Zhang et al., 2016). Indeed, the growth of newly formed bone is limited by the initially available space and the scaffold's degradation rate. Degradation of the material is required for complete bone healing. For example, Seol et al. observed greater bone tissue regeneration with a printed HA/TCP template than with a more slowly degrading printed HA template (Seol et al., 2014). In the present study, the $\mu$-CT parameters did prevented us from detecting the honeycomb PCL-nHA scaffold and thus from evaluating its rate of degradation over time. However, given that the mean \pm SEM thickness of our honeycomb PCL-nHA scaffold was $31 \pm 5 \mu \mathrm{m}$, (Garcia Garcia et al., 2018) we can assume that it did not fill the bone defect (500 $\mu \mathrm{m}$ in thickness). Hence, the scaffold left space 
for bone synthesis, which might have contributed to the rapid bone construction (with a bone volume fraction of more than $80 \%$ after 8 weeks) relative to most of the scaffolds tested in the same model (Chamieh et al., 2016; Chen et al., 2019; Orbay et al., 2017; Son et al., 2013; Wang et al., 2019; Wu et al., 2019; Zhang et al., 2016). Moreover, we did not observe PCL fibers on the histological sections. In the same model, Son et al. (2013) demonstrated the osteoconductive properties of an electrospun PCL/poly(methylmethacrylate) scaffold. At the end point ( 8 weeks), they observed the presence of PCL fibers. We hypothesize that this disparity is due to two parameters: (i) the diameter of the fibers, with a mean value of $2 \mu \mathrm{m}$ in Son et al.'s scaffold, vs. $145 \pm 39 \mathrm{~nm}$ in our scaffold (Garcia Garcia et al., 2018), and (ii) the very slow degradation of poly(methylmethacrylate) (Son et al., 2013), which is slower than that of HA nanoparticles. In view of the results obtained here, we considered that our honeycomb PCL-nHA scaffold provided sufficient support for cell adhesion and colonization and also left sufficient space for bone growth.

Overall, the scaffold's structure, composition and combination with BM-MSCs accelerated the bone regeneration process. However, the scaffold's mechanical properties in vivo remain to be determined. It would be interesting to evaluate the scaffold's bone regeneration capacity in a context of mechanical stress, such as animal models of mandibular or tibia defects.

\section{Conclusion}

We demonstrated the in vitro and in vivo biocompatibility and osteoconductive properties of an electrospun/electrosprayed honeycomb PCL-nHA scaffold. When implanted in a rat calvarial defect, the scaffold exhibited osteoinductive activity and thus promoted new bone formation. The honeycomb PCL-nHA scaffold accelerated the bone regeneration process during the immediate post-implantation period. The combination of honeycomb PCL-nHA scaffold and BM-MSCs significantly improved bone regeneration and bone mineralization in 
particular. This biomimicry-based MSC-seed scaffold appears to be a promising candidate for healing/repairing clinically relevant large craniofacial bone defects.

\section{Conflict of interest}

The authors declare that they have no competing or commercial interests.

\section{Authors' contributions}

SLR, CL, BD, JPM, MN, AGG conceived and designed the experiments. MN and AGG performed the experiments (MN: all animal experiments, AGG: fluorescence and MEB experiments). AGG, FB, AH and GS designed and produced the PCL-nHA honeycomb scaffold. NJ optimized conditions of cells culture with scaffold. AB isolated rat cells from bone marrow. LC performed MSCs phenotyping by flow cytometry. MN and LZ realized $\mu \mathrm{CT}$ acquisitions and analyses. SZA realized histological analysis. MN drafted the manuscript. SLR, CL, JPM, BD, GS, AH and NJ revised the manuscript. All authors approved the final version of the manuscript for submission.

\section{Acknowledgment}

This research was funded by the French government via the "Investments for the Future" program (managed by the National Research Agency; reference: ANR-11-IDEX-0004-02), and by the Region Hauts de France (the INTIM project). We thank Sabine Siegwald for fabricating the micropatterned collectors at the STnano cleanroom facility (University of Strasbourg, Strasbourg, France). We also thank Julie Le Ber and Anaïs Jovelet (PlatAnN animal facility, Jules Verne University of Picardie, Amiens, France) for assistance with animal experiments, and Paulo Marcelo (ICAP cell engineering and protein analysis facility, Jules Verne University of Picardie, Amiens, France) for assistance with $\mu$ CT. A. Hébraud and G. Schlatter thank the financial support of the project ANR MimHeart (ANR-15-CE08-0010). 
Finally, we acknowledge the contribution of the physico-chemical analysis platform at UTC (Frédéric Nadaud and Caroline Lefebvre), for confocal and SEM analysis.

\section{References}

Alford A.I., Kozlorff K.M., Hankenson K.D. (2015). Extracellular matrix networks in bone remodeling. The International Journal of Biochemistry \& Cell Biology, 65, 20-31, https://doi.org/10.1016/j.biocel.2015.05.008.

Baudequin T., Gaut L., Mueller M., Huepkes A., Glasmacher B., Duprez D., Bedoui F., Legallais C. (2017). The Osteogenic and Tenogenic Differentiation Potential of C3H10T1/2 (Mesenchymal Stem Cell Model) Cultured on PCL/PLA Electrospun Scaffolds in the Absence of Specific Differentiation Medium. Materials, 10, e1387. https://doi.org/10.3390/ma10121387.

Borden M., El-Amin S.F., Attawia M., Laurencin C.T. (2003) Structural and human cellular assessment of a novel microsphere-based tissue engineered scaffold for bone repair. Biomaterials, 24, 597-609. https://doi.org/10.1016/S0142-9612(02)00374-5.

Brennan M.A., Renaud A., Gamblin A-L., D’Arros C., Nedellec S., Trichet V., Layrolle P. (2015). 3D cell culture and osteogenic differentiation of human bone marrow stromal cells plated onto jet-sprayed or electrospun micro-fiber scaffolds. Biomed. Mater., 10, 045019. https://doi.org/10.1088/1748-6041/10/4/045019.

Chamieh F., Collignon A-M., Coyac B.R., Lesieur J., Ribes S., Sadoine J., Llorens A., Nicoletti A., Letourneur D., Colombier M-L., Nazjhats S.N., Bouchard P., Chaussain C., Rochefort G.Y. (2016). Accelerated craniofacial bone regeneration through dense collagen 
gel scaffolds seeded with dental pulp stem cells. Scientific Reports, 6:38814. https://doi.org/10.1038/srep38814.

Charoenlarp P., Rajendran A.K., Fujihara R., Kojima T., Nakahama K., Sasaki Y., Akiyoshi K., Takechi M., Iseki S. (2018). The improvement of calvarial bone healing by durable nanogel-crosslinked materials. Journal of Biomaterials Science, Polymer Edition, 29, 18761894. https://doi.org/10.1080/09205063.2018.1517403.

Chen M., Xu Y., Zhang T., Ma Y., Liu J., Yuan B., Chen X., Zhou P., Zhao X., Pang F., Liang W. (2019). Mesenchymal stem cell sheets: a new cell-based strategy for bone repair and regeneration. Biotechnology Letters, 41, 305-311. https://doi.org/10.1007/s10529-019$\underline{02649-7 .}$

Chou J., Hao J., Kuroda S., Ben-Nissan B., Milthopre B., Otsuka M. (2014). Bone regeneration of calvarial defect using marine calcareous-derived beta-tricalcium phosphate macrospheres. Journal of Tissue Engineering, 5. https://doi.org/10.1177/2041731414523441.

Criscenti G., Vasilevich A., Longoni A., de Maria C., van Blitterswijk C.A., Truckenmuller R., Vozzi G., De Boer J., Moroni L. (2017). 3D screening device for the evaluation of cell response to different electrospun microtopographies. Acta Biomaterialia, 55, 310-322, https://doi.org/10.1016/j.actbio.2017.03.049.

Ding X., Yang G., Zhang W., Li G., Lin S., Kaplan D.L., Jiang X. (2017). Increased stem cells delivered using a silk gel/scaffold complex for enhanced bone regeneration. Scientific Reports, 7 :2175. https://doi.org/10.1038/s41598-017-02053-Z.

Dorozhkin S.V. (2010). Bioceramics of calcium orthophosphates. Biomaterials, 31, 14651485. https://doi.org/10.1016/j.biomaterials.2009.11.050. 
Eniwumide J.O., Tanaka M., Nagai N., Morita Y., de Bruijn J., Yamamoto S., Onodera S., Kondo E., Yasuda K., Shimomura M. (2014). The Morphology and Functions of Articular Chondrocytes on a Honeycomb-Patterned Surface. BioMed Research International. https://doi.org/10.1155/2014/710354.

Garcia Garcia A., Hebraud A., Duval J-L., Wittmer C.R., Gaut L., Duprez D., Egles C., Bedoui F., Schlatter G., Legallais C. (2018). Poly(e-caprolactone)/hydroxyapatite 3D honeycomb scaffolds for a cellular microenvironment adapted to maxillofacial-bone reconstruction. ACS Biomater. Sci. Eng., 4, 3317-3326. https://doi.org/10.1021/acsbiomaterials.8b00521.

Jensen J., Tvedesøe C., Rölfing J.H., Foldager G.B., Lysdahl H., Kraft D.C., Chen M., Baas J., Le D.Q., Bünger C.E. (2016). Dental pulp-derived stromal cells exhibit a higher osteogenic potency than bone marrow-derived stromal cells in vitro and in a porcine critical-size bone defect model. SICOT J., 2-16. https://doi.org/10.1051/sicotj/2016004.

Kiernan C.H., Wolvius E.B., Brama P.A.J., Farrell E. (2018). The Immune Response to Allogeneic Differentiated Mesenchymal Stem Cells in the Context of Bone Tissue $\begin{array}{lllll}\text { Engineering. } & \text { Tissue } & \text { Engineering: } & \text { Bart }\end{array}$ https://doi.org/10.1089/ten.teb.2017.0175.

Kwon D.Y., Park J.H., Jang S.H., Park J.Y., Jang J.W., Min B.H., Kim W-D., Lee H.B., Lee J., Kim M.S. (2018). Bone regeneration by means of a three-dimensional printed scaffold in a rat cranial defect. J. Tissue Eng. Regen. Med., 12, 516-528. https://doi.org/10.1002/term.2532.

Lee S-H., Lee K-G., Hwang J-H., Cho Y.S., Lee K-S., Jeong H-J., Park S-H., Park Y., Cho YS., Lee B-Y. (2019). Evaluation of mechanical strength and bone regeneration ability of 3D 
printed kagome-structure scaffold using rabbit calvarial defect model. Materials Science \& Engineering C, 98, 949-959. https://doi.org/10.1016/j.msec.2019.01.050.

Leijten J. and Khademhosseini A. (2016). From Nano to Macro: Multiscale Materials for Improved Stem Cell Culturing and Analysis. Cell Stem Cell, 18, 20-24. https://doi.org/10.1016/j.stem.2015.12.013.

Mankani M.H., Kuznetsov S.A., Fowler B., Kingman A., Robey M. G. (2001). In Vivo Bone Formation by Human Bone Marrow Stromal Cells: Effect of Carrier Particle Size and Shape. Biotechnol Bioeng, 72, 96-107. https://doi.org/10.1002/1097-0290(20010105)72:1<96::aidbit13>3.0.co;2-a.

Nedjari S., Eap S., Hebraud A., Wittmer C.R., Benkirane-Jessel N., Schlatter G. (2014). Electrospun Honeycomb as Nests for Controlled Osteoblast Spatial Organization. Macromol. Biosci., 14, 1580-1589. https://doi.org/10.1002/mabi.201400226.

Orbay H., Busse B., Leach J.K., Sahar D. E. (2017). The Effects of Adipose-Derived Stem Cells Differentiated Into Endothelial Cells and Osteoblasts on Healing of Critical Size Calvarial Defects. Journal of Craniofacial Surgery, 28, 1874-1879. https://doi.org/10.1097/SCS.0000000000003910.

Oryan A., Kamali A., Moshiri A., Baghaban Eslaminejad M. (2017). Role of Mesenchymal Stem Cells in Bone Regenerative Medicine: What Is the Evidence ?. Cells Tissues Organs, 204, 59-83. https://doi.org/10.1159/000469704.

Pae H-C., Kang J-H., Cha J-K., Lee J-S., Paik J-W., Jung U-W., Kim B-H., Choi S-H. (2019). 3D-printed polycaprolactone scaffold mixed with $\beta$-tricalcium phosphate as a bone regenerative material in rabbit calvarial defects. J Biomed Mater Res B Appl Biomater., 4, 1254-1263. https://doi.org/10.1002/jbm.b.34218. 
Rao S.H., Harini B., Shadamarshan R. P.K., Balagangadharan K., Selvamurugan N. (2018). Natural and Synthetic Polymers/Bioceramics/Bioactive Compounds-mediated Cell Signaling in Bone Tissue Engineering. Int J Biol Macromol., 110, 88-96. https://doi.org/ 10.1016/j.ijbiomac.2017.09.029.

Seol Y-J., Park J.K., Jung J.W., Jang J., Girdhari R., Kim S.W., Cho D-W. (2014). Improvement of bone regeneration capacity of ceramic scaffolds by accerelated release of their calcium ions. Tissue Eng. Part A., 20, 2840-2849. https://doi.org/10.1089/ten.TEA.2012.0726.

Shao X., Goh J.C., Hutmacher D.W., Lee E.H., Zigang G. (2006). Repair of Large Articular Osteochondral Defects Using Hybrid Scaffolds and Bone Marrow-Derived Mesenchymal Stem Cells in a Rabbit Model. Tissue Engineering, 12, 1539-1551. https://doi.org/10.1089/ten.2006.12.1539.

Shor L., Güçeri S., Chang R., Gordon J., Kang Q., Hartsock L., An Y., Sun W. (2009). Precision extruding deposition (PED) fabrication of polycaprolactone (PCL) scaffolds for bone tissue engineering. Biofabrication, 1, 015003. https://doi.org/10.1088/1758$\underline{5082 / 1 / 1 / 015003 .}$

Son S-R., Linh N-T.B., Yang H-M., Lee B-T. (2013). In vitro and in vivo evaluation of electrospun PCL/PMMA fibrous scaffolds for bone regeneration. Sci. Technol. Adv. Mater., 14, 015009. https://doi.org/10.1088/1468-6996/14/1/015009.

Sousa B.R., Parreira R.C., Fonseca E.A., Amaya M.J., Tonelli F.M.P., Lacerda S.M.S.N., Lalwani P., Santos A.K., Gomes K.N., Ulrich H., Kihara A.H., Resende R.R. (2014). Human adult stem cells from diverse origins: an overview from multiparametric immunophenotyping to clinical applications. Cytometry part A, 85, 43-77. https://doi.org/10.1002/cyto.a.22402. 
Wang Y., Cui W., Zhao X., Wen S., Sun Y., Han J., Zhang H. (2019). Bone remodelinginspired dual delivery electrospun nanofibers for promoting bone regeneration. Nanoscale, 11, 60-71. https://doi.org/10.1039/c8nr07329e.

Wittmer C., Heb́raud A., Nedjari S., Schlatter G. (2014). Well-Organized 3D Nanofibrous Composite Constructs Using Cooperative Effects between Electrospinning and Electrospraying. Polymer, 55, 5781-5787. https://doi.org/10.1016/j.polymer.2014.08.044.

Wu J., Cao L., Liu Y., Zheng A., Jiao D., Zeng D., Wang X., Kaplan D.L., Jiang X. (2019). Functionalization of silk fibroin electrospun scaffolds via BMSC affinity peptide grafting through oxidative self-polymerization of dopamine for bone regeneration. ACS Appl. Mater. Interfaces, 11, 8878-8895. https://doi.org/10.1021/acsami.8b22123.

Xu F.J., Wang Z.H., Yang W.T. (2010). Surface functionalization of polycaprolactone films via surface-initiated atom transfer radical polymerization for covalently coupling celladhesive biomolecules. Biomaterials, 31, 3139-3147. https://doi.org/10.1016/j.biomaterials.2010.01.032.

Yagyuu T., Kirita T., Hattori K., Tadokoro M., Ohgushi H. (2012). Unique and reliable rat model for the assessment of cell therapy: bone union in the rat mandibular symphysis using the bone marrow stromal cells. J Tissue Eng regen Med., 9, 276-285. https://doi.org/10.1002/term.1674.

Yang F., Murugan R., Wang S., Ramakrishna S. (2005). Electrospinning of nano/micro scale poly(L-lactic acid) aligned fibers and their potential in neural tissue engineering. Biomaterials, 26, 2603-2610. https://doi.org/10.1016/j.biomaterials.2004.06.051. 
Yao C-H., Yang S-P., Chen Y-S., Chen K-Y. (2019). Electrospun Poly(-glutamic acid)/Tricalcium Phosphate Composite Fibrous Mats for Bone Regeneration. Polymers, 11, E227. https://doi.org/10.3390/polym11020227.

Ye K., Liu D., Kuang H., Cai J., Chen W., Sun B., Xia L., Fang B., Morsi Y., Mo X. (2019). Three-dimensional electrospun nanofibrous scaffolds displaying bone morphogenetic protein2-derived peptides for the promotion of osteogenic differentiation of stem cells and bone regeneration. Journal of Colloid and Interface Science, 534, 625-636. https://doi.org/10.1016/j.jcis.2018.09.071.

Zhang H., Mao X., Du Z., Jiang W., Han X., Zhao D., Han D., Li Q. (2016). Three dimensional printed macroporous polylactic acid/hydroxyapatite composite scaffolds for promoting bone formation in a critical-size rat calvarial defect model. J. Sci. Technol. Adv. Mater., 17, 136-148. https://doi.org/10.1080/14686996.2016.1145532.

Zhang Z-Y., Teoh S-H., Hui J.H.P., Fisk N.M., Choolani M., Chan J.K.Y. (2012). The potential of human fetal mesenchymal stem cells for off-the-shelf bone tissue engineering application. Biomaterials, 33, 2656-2672. https://doi.org/10.1016/j.biomaterials.2011.12.025. 


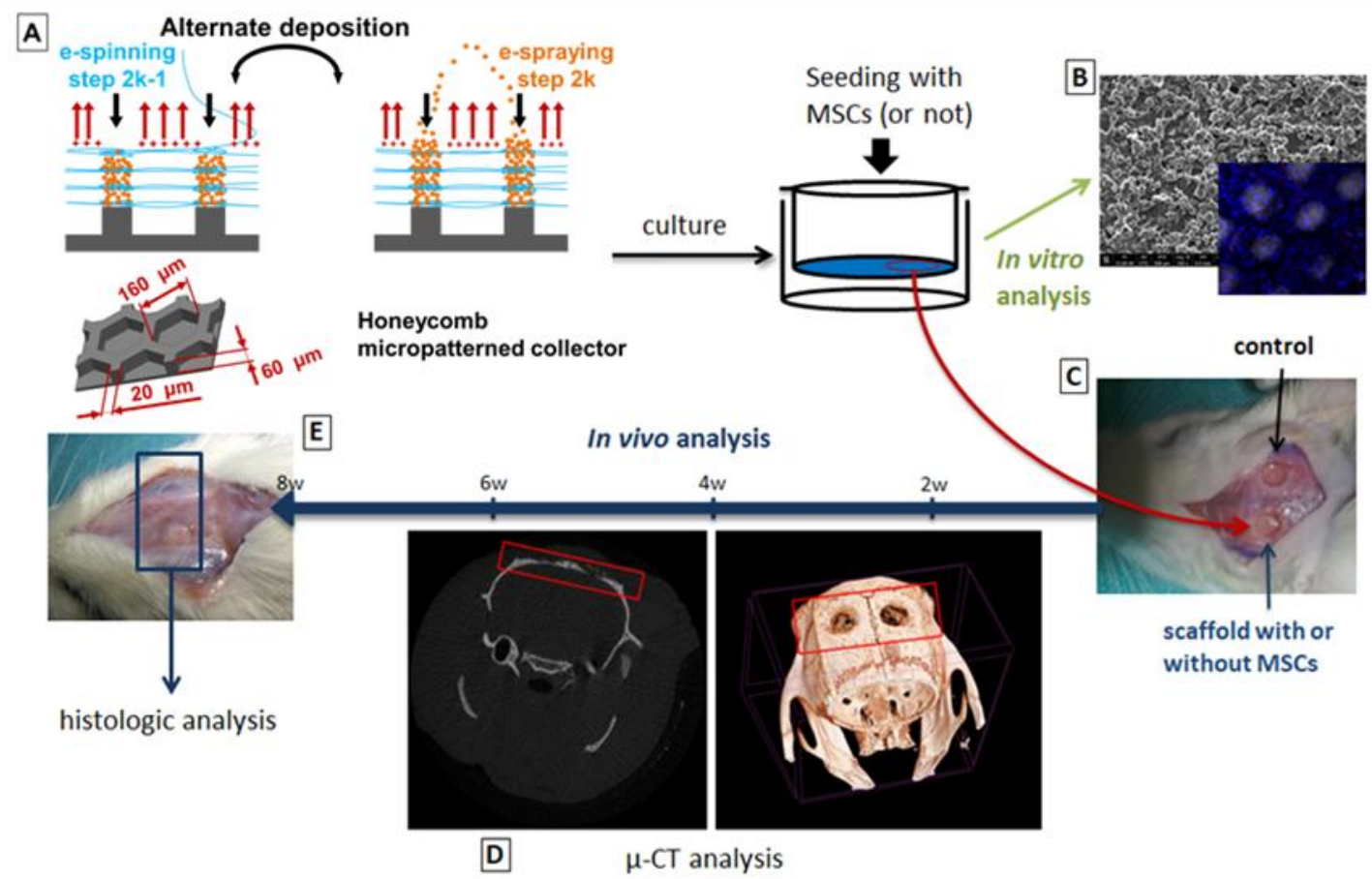

Figure 1. The experimental protocol. The honeycomb PCL-nHA scaffold was produced by alternating electrospun PCL fiber layers (steps $2 \mathrm{k}-1$ for $\mathrm{k}=1$ to 7 ) and electrosprayed HA nanoparticle layers (steps $2 \mathrm{k}$ for $\mathrm{k}=1$ to 6 ) (A). After the cells had been cultured for $24 \mathrm{~h}$, the MSCs' adhesion and viability were analyzed using fluorescence microscopy and scanning electron microscopy (SEM) (B). Honeycomb PCL-nHA scaffolds with or without BM-MSCs were engrafted into a calvarial bone lesion on one side of the body; the contralateral lesion remained empty and served as a control $(\mathrm{C})$. Bone regeneration was analyzed using $\mu \mathrm{CT}$ after 2, 4, 6 and 8 weeks of implantation (D). The red frames delimit the areas analyzed (cf. Fig 4). After 8 weeks, the part of the skull containing the defects was recovered for histological assessment (E).
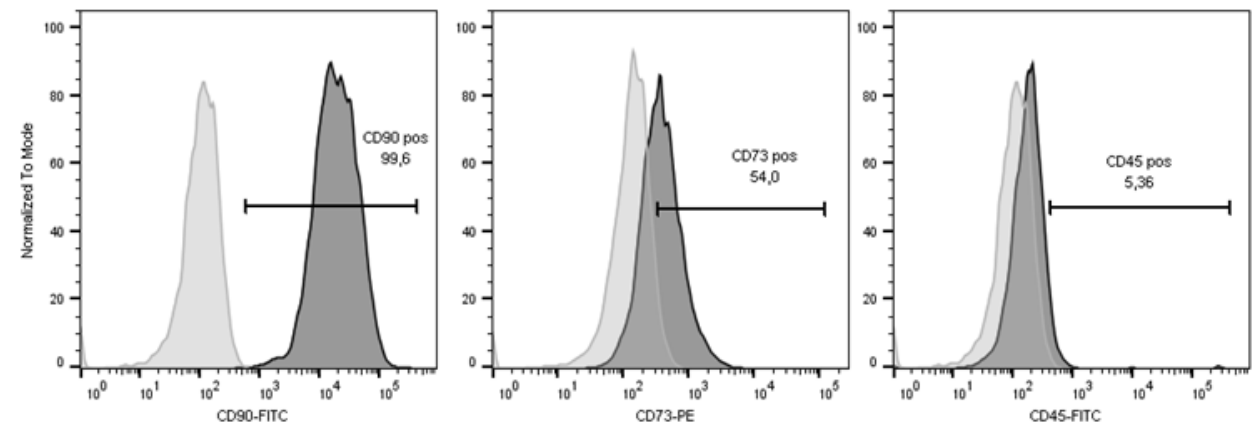

Figure 2. Phenotyping of rat BM-MSCs. The expression of three cell surface markers associated with the MSC phenotype in humans (CD90, CD73, and CD45) was investigated 
using flow cytometry. The dark grey histograms indicate cells that stained positive. Isotypematched controls are overlaid as light grey histograms.
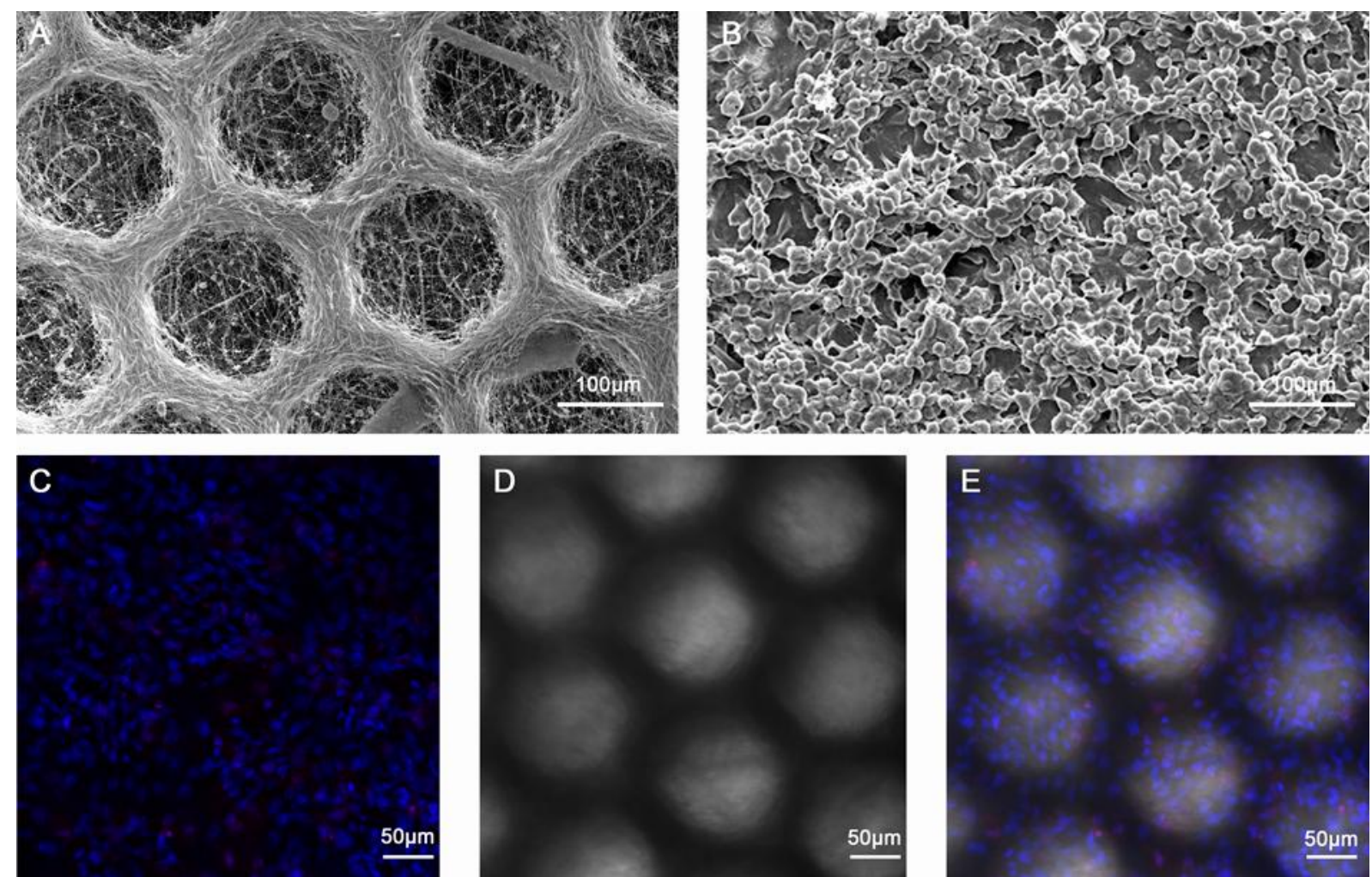

Figure 3. Spatial distribution and viability of BM-MSCs seeded on a scaffold before implantation. Scanning electron micrographs of a honeycomb PCL-nHA scaffold with no cells (A) and one seeded with BM-MSCs (B). Scale bar in A and B: $100 \mu \mathrm{m}$. Fluorescence staining of BM-MSCs with Hoechst 33342 (blue) and EthD-1 (red) on a scaffold (C), the transmitted light image (D), and the merged images (E). Scale bar in C-E: $50 \mu \mathrm{m}$. 


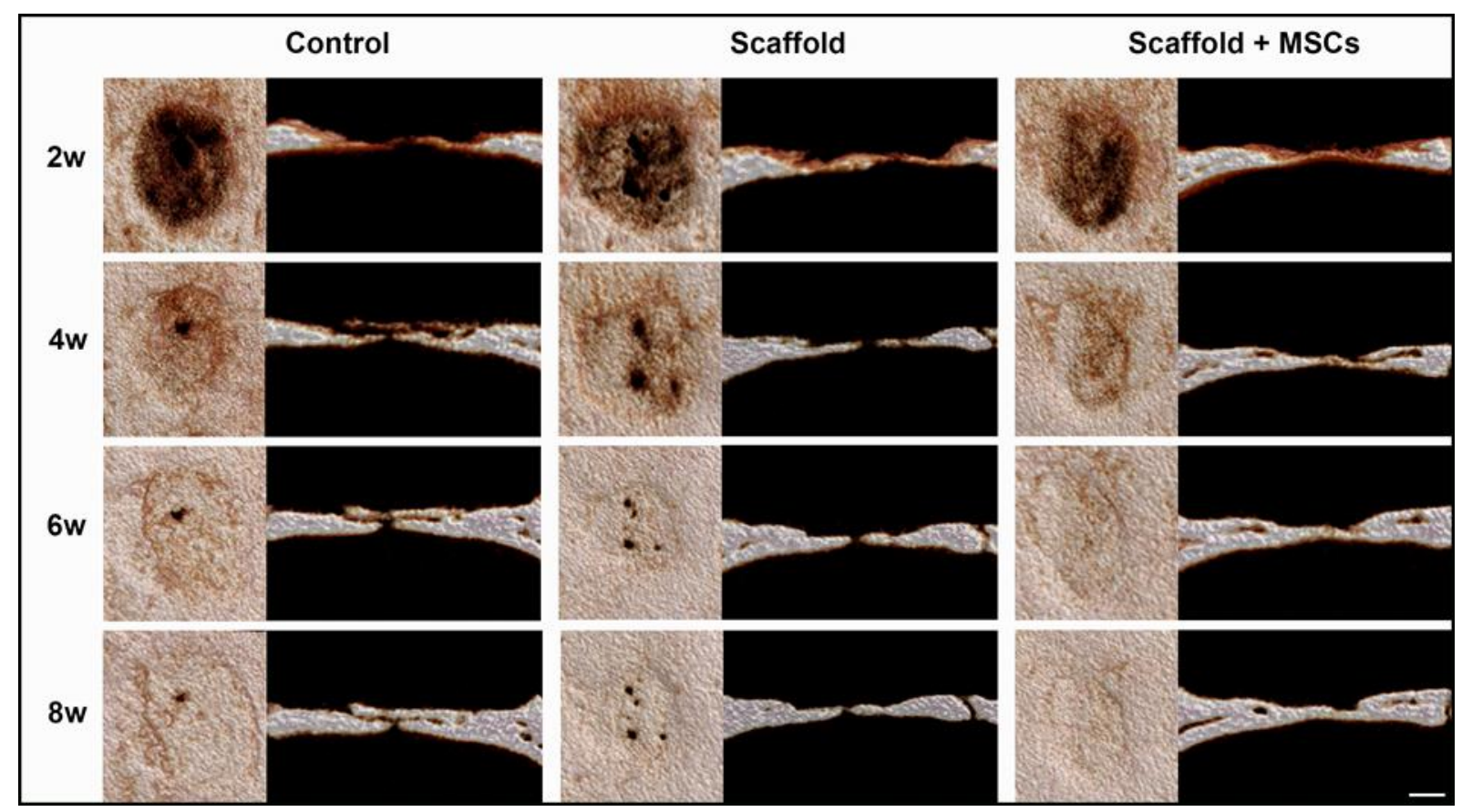

Figure 4. Micro-CT of calvarial defects in the control, scaffold and scaffold+MSCs groups at 2, 4, 6 and 8 weeks post-implantation. For each condition, the left panel shows a sagittal view of the defect and the right panel shows a coronal view. Scale bar: $1 \mathrm{~mm}$.
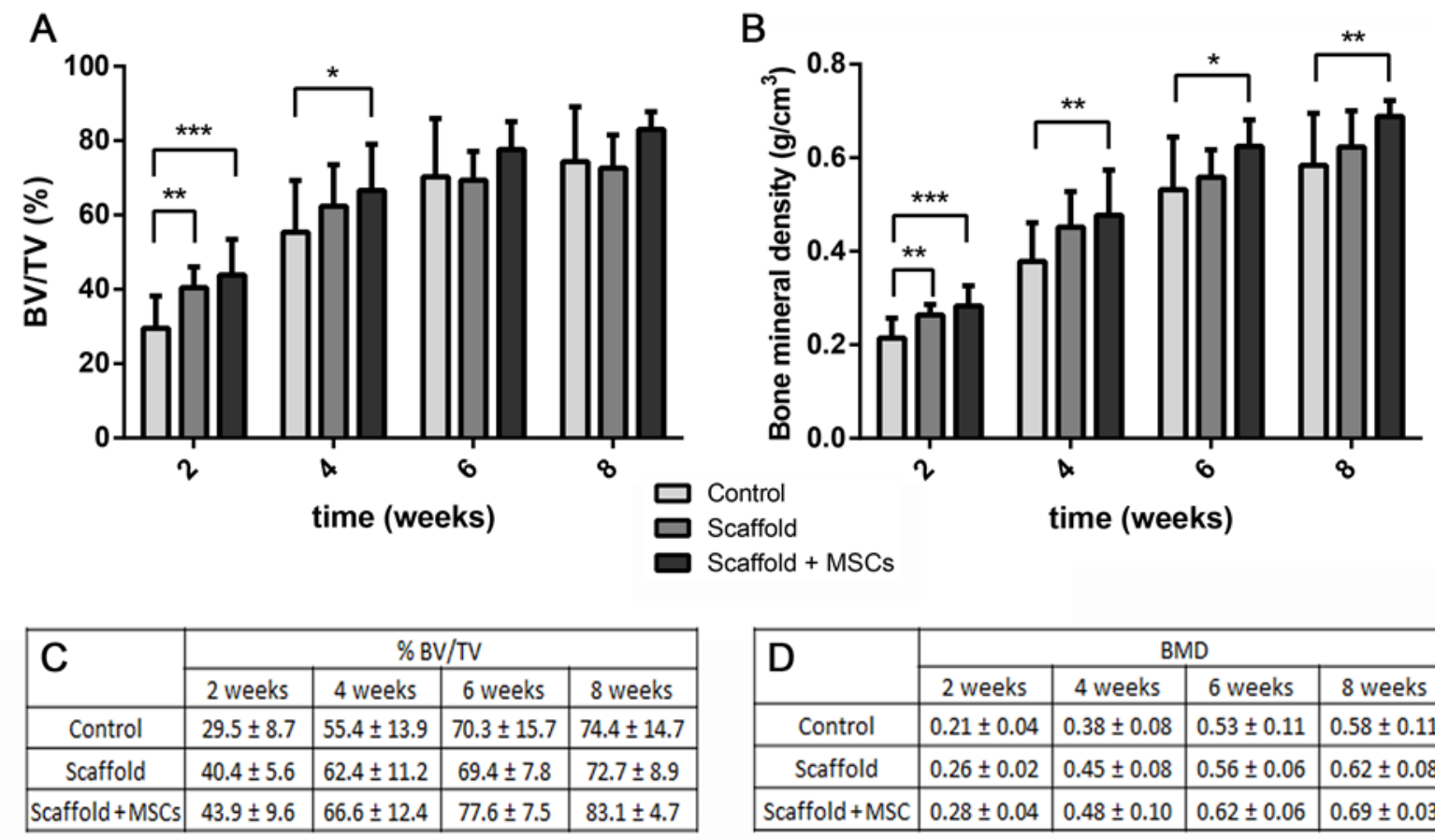

Figure 5. Quantitative analysis of bone generation in the defect at 2, 4, 6 and 8 weeks, as evaluated by the bone volume fraction (the BV/TV ratio in \%) (A, B) and the BMD (in $\mathrm{g} / \mathrm{cm}^{2}$ ) (C, D). *p $<0.05 ; * * p<0.01 ; * *$ p $<0.001$. 


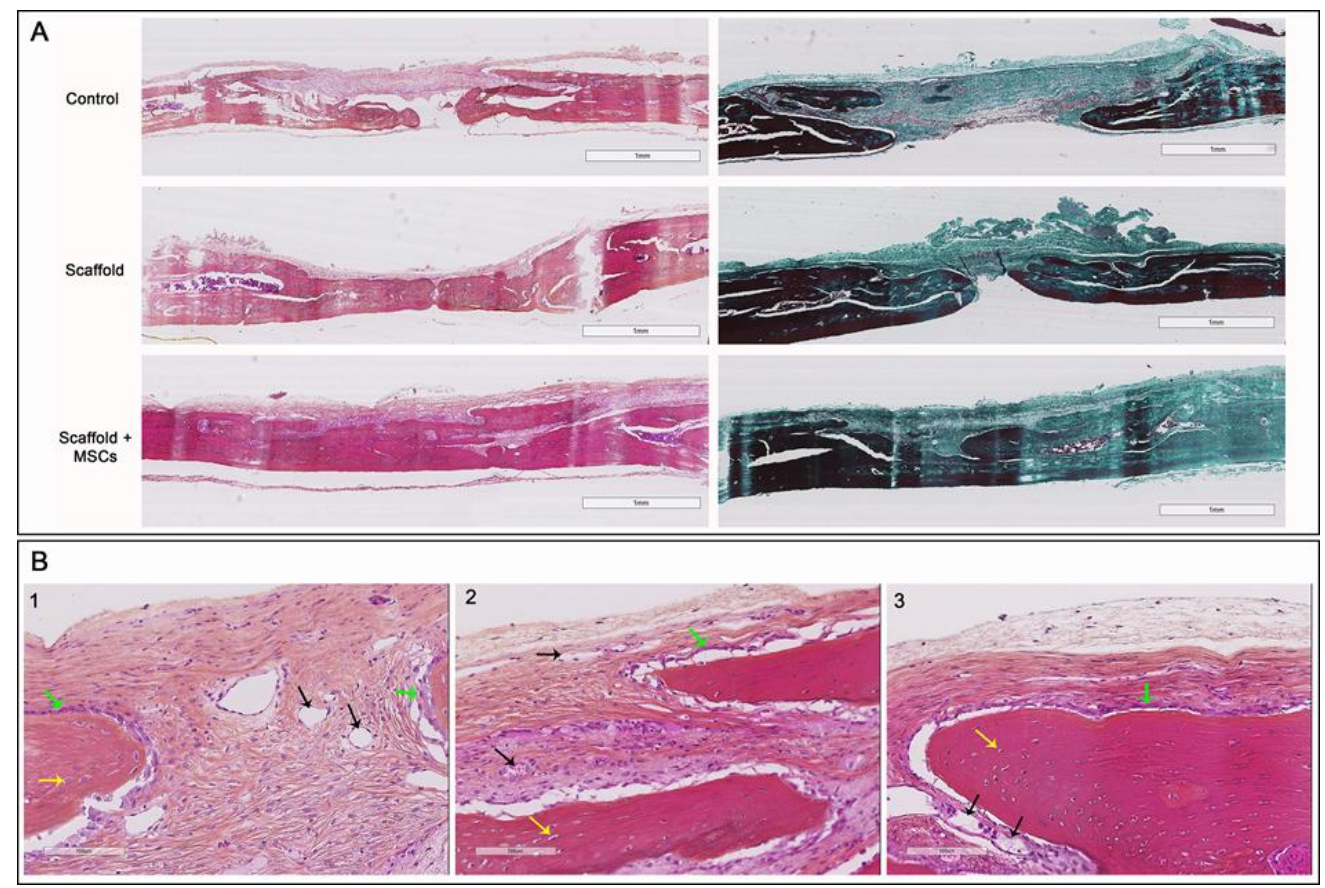

Figure 6. Representative histologic images of calvarial defects eight weeks after implantation in the control, scaffold and scaffold+MSCs groups (H\&E staining on the left and Masson's trichrome staining on the right) (A). Scale bar $=1 \mathrm{~mm}$. A higher-magnification view of $\mathrm{H} \& \mathrm{E}$ stained sections from the control (1), scaffold-only (2) and scaffold+MSCs groups (3) (B), black arrow: vessel; green arrow: osteoblast; yellow arrow: osteocyte. Scale bar $=100 \mu \mathrm{m}$. 